\title{
Une méthode basée sur les polices Multiple Master pour mettre à l'échelle des glyphes sans en changer les caractéristiques de trait
}

\author{
Tim Ahrens \\ 290, Abingdon road \\ Oxford OX1 4TE, Angleterre \\ mail@tim-ahrens.de
}

Traduction française de Patrick Andries (patrick@ hapax. qc. ca)

\begin{abstract}
RÉSUMÉ. Cet article présente la "Mise à échelle compensatrice », une méthode d'avant-garde qui permet de modifier automatiquement les dimensions des glyphes. Pour les polices Multiples Masters ayant un axe "épaisseur", cette information sur la graisse permet de compenser les changements d'épaisseur de trait dus à un changement d'échelle. Elle peut également servir à créer de véritables petites capitales, des minuscules cyrilliques et-puisque les échelles verticale et horizontale peuvent être ajustées indépendamment l'une de l'autre - de vraies polices étroites. La comparaison avec des styles de caractères existants montre que le résultat du modèle est relativement proche de celui des vrais oeils. Contrairement aux formats de données "intelligents », le modèle présenté ci-dessous est une méthode de traitement de formes préexistantes qui n'est pas tributaire d'une technique de police particulière. Le résultat produit par cette méthode a le même format que celui des données fournies en entrée et il peut donc être réutilisé par d'autres outils. Cette méthode peut donc être l'un des modules d'une chaîne de dessin de caractères, plutôt qu'un outil autonome.

ABSTRACT. This article presents Compensating Scaling, an advanced method for changing the dimensions of glyphs. Working with MM fonts that have a weight axis, the "boldness" information is used to compensate for changes in stroke weight caused by the scaling. The method helps to create true small caps, Cyrillic lowercase and - since the horizontal and vertical scale factors can be chosen independently - even true condensed fonts. Comparisons to existing typefaces show that the output generated by the model is rather close to that of the true glyphs. In contrast to "intelligent" data formats the model suggested here is a technology-independent formula for the processing of pre-existing shapes. The output has the same format as the input
\end{abstract}

DN - 9/2006. Fontes numériques, pages 133 à 165 
134

DN - 9/2006. Fontes numériques

(1)

and can be subsequently processed, making the method a tool for the automation of a specific design step rather than a stand-alone technology.

MOTS-CĹS : Polices Multiple Master, archétype, création de polices, petites capitales, cyrillique, autoniatisation, FontLab

KEYWORDS: Multiple Master, typeface design, small caps, Cyrillic, automation, FontLab 


\section{Objectifs}

Les polices numériques ont connu diverses innovations et avancées depuis leurs débuts il y a plus de quarante ans et les progrès se poursuivent. L'apparition d'OpenType et la prise en charge croissante d'Unicode constituent deux améliorations révolutionnaires dans le traitement des glyphes. En revanche, le domaine de la forme des glyphes semble être en panne depuis plusieurs années. Les années 1970, 1980 et le début des années 1990 ont vu l'apparition de plus de 150 formats de police (Karow, 1994, p. 22) et la mise au point de plusieurs méthodes mathématiques qui suscitèrent des controverses. Après cette phase d'innovations et de spéculations, les formats TrueType et PostScript, tous deux définis à l'aide de contours, « ont remporté la compétition » (Jackowski, 1999). Après l'abandon de la technique Multiple Master ${ }^{1}$ par Adobe, il semble que la description statique de glyphe, précisée directement par le concepteur de polices sans faire appel à des expressions mathématiques paramétrées, soit acceptée par tous comme le seul système pratique de production de documents imprimés de qualité. Les mathématiciens ne paraissent pas oser explorer d'autres méthodes de définition des glyphes qui d'ailleurs ne semblent être souhaitées ni par les concepteurs ni par les typographes.

Cet article reprend le débat sur les modèles mathématiques utilisés dans la description de la forme des glyphes. Contrairement aux formats «intelligents » comme METAFONT ou Intellifont, le modèle proposé ici ne sert pas à la création de formes. Il s'agit simplement d'un outil de traitement de glyphes préexistants. Il produit en sortie des données de même format qu'en entrée, celles-ci peuvent être traitées par la suite, pour corriger manuellement les défauts qui y resteraient. Il ne s'agit donc pas d'un outil autonome, mais d'une méthode qui peut être utilisée par les concepteurs et qui permet d'automatiser une étape particulière de la conception. Toutefois, on peut encore en imaginer d'autres utilisations comme le post-traitement des polices par les moteurs de rendu ou comme l'analyse des dessins de police.

Beaucoup de glyphes dérivent d'autres glyphes et ne différent que par leur taille. Toutefois, modifier de façon proportionnelle l'épaisseur d'un trait, lors d'un changement d'échelle d'un glyphe, entraîne des altérations inacceptables des caractéristiques de ce glyphe (voir figure 1).

\section{Les Vraies Petites Capitales Ne Sont Pas Que Des Majuscules Rétrécies Qu'on Aurait Lavées À Trop Haute Température}

Figure 1. Changer d'échelle modifie l'épaisseur des traits; texte traduit de (Rögener et al., 1995)

1. Note du traducteur : pour une description des polices MultiMaster ou «polices accordéon », voir l'excellent ouvrage de Yannis Haralambous (2004), Fontes \& Codage. Voir plus particulièrement les pages 460 à 463 et 668 à 674. Nous en adoptons la proposition d' "archétype » comme traduction en français du terme anglais master. 
$\mathrm{Si}$ on ne désire pas créer des glyphes à partir de rien, on peut imaginer deux manières de les produire à partir de caractères existants. L'une consiste à changer la taille en déplaçant uniquement des portions du contour plutôt que d'exercer la mise à l'échelle sur toutes les coordonnées de chaque point. Cette opération peut s'effectuer à la main (Hudson, 2005) ou à l'aide d'un programme informatique intelligent (André et al., 1994).

Une autre méthode consiste à effectuer une mise à l'échelle géométrique et à corriger ensuite la force des traits. Ceci se fait habituellement à la main. Comme nous le montrerons ici, ceci peut également se faire à l'aide d'un modèle mathématique. On trouvera ci-dessous la description d'un tel modèle et quelques essais effectués sur celui-ci. On règle l'épaisseur de trait à l'aide d'un second archétype d'une force différente qui contient l'information de graisse nécessaire que l'on peut ajouter ou soustraire au besoin.

Les Multiple Master permettent de décrire les contours d'un glyphes sous une forme numérique et de manipuler sans difficulté les formes correspondantes. Bien que ce format de police soit désormais abandonné en tant que format final, il est encore souvent utilisé par les outils de création de polices et il est de mieux en mieux accepté par les éditeurs de polices.

Notre modèle - que nous appellerons «à échelle compensée »-pourra servir tant au concepteur novice qu'à l'expert. Ce dernier épargnera du temps, alors que le novice pourra de la sorte améliorer le dessin, car l'outil augmente la cohérence au sein des polices ainsi que des familles de polices. Tout comme une scie circulaire ne peut fabriquer toute seule des meubles et n'améliore peut-être même pas le produit fini de l'artisan, elle épargne, toutefois, beaucoup de temps. Elle effectue très bien certains aspects du travail sans empêcher une finition ultérieure.

Cet article s'articule de la façon suivante : la section 2 présente le modèle. Ses formules, partant d'un principe d'abord simple, s'améliorent au fur et à mesure que de nouveaux aspects sont pris en compte. La section 3 vise à mettre le modèle à l'épreuve. En plus de le comparer directement au module Ek, les résultats de notre modèle sont comparés, sur des polices connues, pour les versions étroites, les petites capitales et les minuscules cyrilliques. Cette comparaison permettra de déterminer si les résultats du modèle mathématique se rapprochent suffisamment des polices faites main pour qu'ils puissent faire gagner du temps et servir de base à une finition ultérieure de la part du concepteur. Comparer les formes automatiques aux formes «réelles » permet de tester non pas le potentiel créatif de la méthode, mais de démontrer son utilité analytique. La comparaison, d'une part, des glyphes mis à l'échelle automatiquement et considérés comme des variations sans changement de style de leur base commune et, d'autre part, leurs versions conçues à la main révèle des modifications de la part du concepteur parfois insoupçonnées. La section 4 décrit en détail les différentes applications de cette méthode. Enfin, la section 5 signale quelques propriétés singulières de notre démarche et tire les conclusions de notre étude. 


\section{La méthode}

Si on réduit optiquement un glyphe, ses traits s'amincissent également. À l'aide d'un second archétype plus gras, il est possible d'en augmenter la graisse. La méthode présentée ci-dessous n'est ni un procédé ni un algorithme, il ne s'agit que d'une équation, pour les coordonnées $x$ et $y$, qui traite la forme dans une seule étape. Toutefois, à titre d'illustration, on expliquera certaines étapes comme si elles formaient une suite d'opérations successives.

Théoriquement, la compensation de perte d'épaisseur de trait a lieu avant la réduction d'échelle. En d'autres termes, on met à l'échelle une instance intermédiaire interpolation entre la forme normale et grasse (fig. 2). Cette instance est calculable.

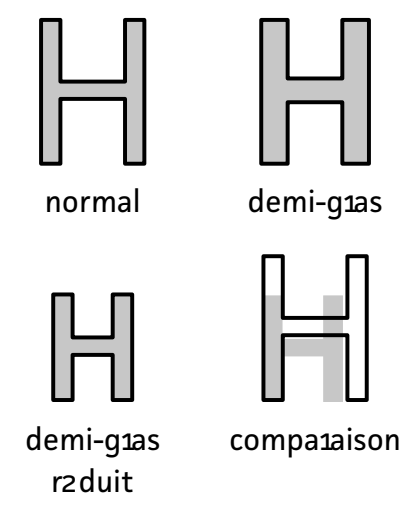

Figure 2. Compensation de la perte de graisse

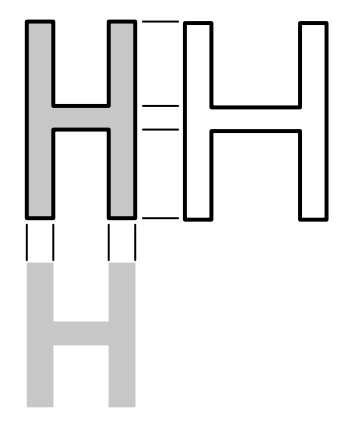

Figure 3. Interpolation anisotrope

Les versions étroites se créent en n'appliquant que les changements horizontaux, ce qu'on nomme une interpolation anisotrope. La valeur en $x$ de chaque point correspond à la version réduite demi-grasse alors que les valeurs en $y$ demeurent celles de la version normale (fig. 3 ).

\subsection{Première approche simple}

Faisons l'hypothèse qu'un glyphe de graisse normale doit être réduit par un facteur d'échelle $f$ tout en compensant la perte d'épaisseur de traits.

À cet effet, on construit une interpolation entre l'archétype normal $\left(x_{n}, y_{n}\right)$ et l'archétype gras $\left(x_{g}, y_{g}\right)$ avec un facteur d'interpolation $q$.

$$
\begin{aligned}
& \left.y=f\left[q y_{n}+(1-q) y_{g}\right)\right] \\
& \left.x=f\left[q x_{n}+(1-q) x_{g}\right)\right]
\end{aligned}
$$


On trouve le facteur $q$ en calculant l'épaisseur de trait du résultat final. Soit $n$ l'épaisseur de trait typique d'une police de graisse normale et $r$ le rapport des épaisseurs du gras et du normal; $r n$ est alors la graisse de l'archétype gras. Comme la graisse doit rester constante, l'épaisseur du trait d'origine est égal à l'épaisseur du trait final.

$$
n=f[q n+(1-q) r n]
$$

d'où

$$
q=\frac{1 / f-r}{1-r}
$$

On peut tout de suite éliminer l'épaisseur de trait $n$, ce qui démontre que le modèle ne dépend pas de l'épaisseur des traits et qu'il ne tente pas de les prendre en compte. La notion d'épaisseur de trait n'est qu'un élément passager du raisonnement. Seul $r$, le rapport entre les épaisseurs grasse et normale typiques, intervient dans le traitement.

Les formules [1] et [3] définissent la forme finale. On pourrait n'en faire qu'une seule équation, mais cela n'est pas nécessaire ici. Ces formules ne dépendent pas des courbes de Bézier, on peut les utiliser avec n'importe quel format pour peu qu'il existe deux ensembles de données correspondants.

\subsection{Réglage plus fin de l'épaisseur de traits}

Il n'est guère souvent souhaitable de compenser complètement l'épaisseur de trait. Ainsi, les petites capitales sont-elles habituellement légèrement plus maigres que les majuscules. C'est pourquoi on introduit un facteur d'échelle de traits $t$ : sa valeur se situe entre 0 pour une compensation complète, comme ci-dessus, et 1 pour aucune compensation, en d'autres termes une simple mise à l'échelle géométrique de la forme normale. On définit la valeur $t$ de telle sorte que l'épaisseur de trait est modifiée par le facteur $f^{t} ; f^{0}=1$ signifie que l'épaisseur de trait ne doit pas changer et $f^{1}=f$ signifie que le trait est mis à l'échelle de la même manière que le reste de la forme. On modifie les équations [2] et [3] en conséquence :

$$
\begin{aligned}
& f^{n} t=f[q n+(1-q) r n] \\
& q=\frac{f^{t-1}-b}{1-b}
\end{aligned}
$$


La définition abstraite du paramètre permet au concepteur de choisir, avec un minimum d'expérience, la valeur de manière intuitive. En outre, si on ajuste le facteur d'échelle - par exemple alors qu'on essaie différents corps de petites capitales - le facteur d'échelle des traits peut demeurer inchangé.

\subsection{Mise à l'échelle anisotrope}

Dans la plupart des cas, les facteurs d'échelle horizontale et verticale doivent être différents. La solution mathématique est des plus simples : on définit $f_{x}$ et $f_{y}$ comme des facteurs d'échelle indépendants pour les directions $x$ et $y$, on définit également des valeurs indépendantes $q_{x}$ et $q_{y}$ pour les interpolations horizontale et verticale :

$$
\begin{aligned}
& y=f_{y}\left[q_{y} y_{n}+\left(1-q_{y}\right) y_{g}\right] \\
& x=f_{x}\left[q_{x} x_{n}+\left(1-q_{x}\right) x_{g}\right] \\
& q_{y}=\frac{f_{y}^{t-1}-r}{1-r} \\
& q_{x}=\frac{f_{x}^{t-1}-r}{1-r}
\end{aligned}
$$

\subsection{Prise en compte des angles d'italique}

Si on fait subir à un glyphe une transformation anisotrope, l'angle d'italique change. Pour éviter cet effet, on redresse d'abord le glyphe vers la gauche avant sa transformation puis vers la droite après celle-ci (fig. 4).

$$
d \rightarrow d \rightarrow d \rightarrow d
$$

Figure 4. Ordre naïf du traitement des italiques

Les étapes de la figure 4 ne sont qu'imaginaires car en fait ces opérations s'effectuent simultanément, en une seule équation :

$$
\begin{aligned}
& y=f_{y}\left[q_{y} y_{n}+\left(1-q_{y}\right) y_{g}\right] \\
& x=f_{x}\left[q_{x}\left(x_{n}-y_{r} i\right)+\left(1-q_{x}\right)\left(x_{g}-y_{g} i\right)\right]+y i
\end{aligned}
$$


où $i$ est la tangente de l'angle d'italique. Dans la formule [6], $x_{n}$ et $x_{g}$ sont modifiés en leur soustrayant respectivement $y_{n} i$ et $n y_{g} i$ qui représentent l'inclinaison vers la gauche. Le terme yi permet d'incliner à nouveau le glyphe selon son angle d'origine.

Ceci produit une légère inclinaison des tangentes de Bézier qui définissent d'habitude un glyphe. Cela pose, selon la technique utilisée, un problème qui peut être résolu à l'aide de méthodes appropriées qui sortent cependant du cadre des équations mathématiques exposées ici.

Si l'on souhaite modifier l'angle d'italique, il suffit d'ajuster la valeur $i$.

\subsection{Corps optiques}

Si la police comprend un axe de corps optique, il nous est alors possible de régler l'épaisseur des déliés et des fûts. Au lieu de deux archétypes, on en aura quatre, chacun avec une épaisseur typique de délié et une épaisseur principale de fût. Les coordonnées des quatre archétypes seront $\left(x_{n}, y_{n}\right)$ pour l'épaisseur normale de texte, $\left(x_{g}, y_{g}\right)$ pour le gras, $\left(x_{a n}, y_{a n}\right)$ pour l'épaisseur normale sur écran et $\left(x_{a g}, y_{a g}\right)$ pour le gras sur écran. Les facteurs d'interpolation deviennent $q$ pour l'axe de graisse, et $p$ pour le corps optique :

$$
\begin{aligned}
y= & f_{y}\left[p_{y}\left(q_{y} y_{a n}+\left(1-q_{y}\right) y_{a g}\right)+\left(1-p_{y}\right)\left(q_{y} y_{n}+\left(1-q_{y}\right) y_{g}\right)\right] \\
x= & f_{x}\left[p _ { x } \left(q_{x}\left(x_{a n}-y_{a n} i\right)+\left(1-q_{x}\right)\left(x_{a g}-y_{a g} i\right)\right.\right. \\
& +\left(1-p_{x}\right)\left(q_{x}\left(x_{n}-y_{n} i\right)+\left(1-q_{x}\right)\left(x_{g}-y_{g} i\right)\right]+y i
\end{aligned}
$$

On détermine $p$ et $q$ à l'aide d'une équation similaire à [4]. Soit $n$ l'épaisseur typique de trait des fûts de graisse normale, et $r$ le rapport des épaisseurs du gras et du normal ; $r n$ est alors l'épaisseur du gras. Pour les déliés, l'épaisseur de trait typique de graisse normale peut être $n$ et l'épaisseur de graisse de l'archétype gras peut être $r n$.

On calcule l'épaisseur de fût et de délié de la manière suivante :

$$
\begin{aligned}
& f^{t} n=f\left[p\left(q n_{a}+(1-q) r_{a} n_{a}\right)+(1-p)(q r+(1-q) r n)\right] \\
& f^{t} r=f\left[p\left(q n_{a}+(1-q) r_{a} n_{a}\right)+(1-p)(q n+(1-q) r n)\right]
\end{aligned}
$$

La solution de ces deux équations à deux inconnues ( $p$ et $q$ ) nécessite des rudiments de mathématiques. Comme les équations finales sont très longues, nous ne les présenterons pas ici ; toutefois, le calcul de $p$ et $q$ s'effectue facilement à l'aide d'un ordinateur. 


\subsection{Réduction de l'archétype gras et agrandissement}

Afin de simplifier la discussion, nous avons fait jusqu'à présent l'hypothèse que nous réduisions la taille de l'archétype le plus maigre. Néanmoins, il n'est pas nécessaire de modifier les formules ci-dessus dans les autres cas. Ainsi, si l'on désire réduire l'archétype gras, on peut «prétendre » qu'il s'agit de l'archétype «normal », l'épaisseur de trait de l'autre archétype est toujours définie à l'aide de $r n$ où le rapport de graisse $r<1$ cette fois. Injecté dans [3], on trouve un facteur d'interpolation $q>1$, autrement dit une extrapolation.

Si on agrandit l'archétype le plus maigre, on a donc $f>1$, ceci signifie que $q$ devient négatif, ce qui mène encore une fois à une extrapolation. Si on agrandit l'archétype gras, on obtient une interpolation avec $0<q<1$.

\subsection{Espacement et crénage}

On ne calcule pas explicitement l'approche gauche, il s'agit de la valeur en $x$ du nœud le plus à gauche. La chasse du glyphe se calcule à partir des chasses des deux archétypes à l'aide d'équations similaires à [6] ou [9]. Cela produit un espacement harmonieux et constant comme l'illustre l'exercice ci-dessous (fig. 5).

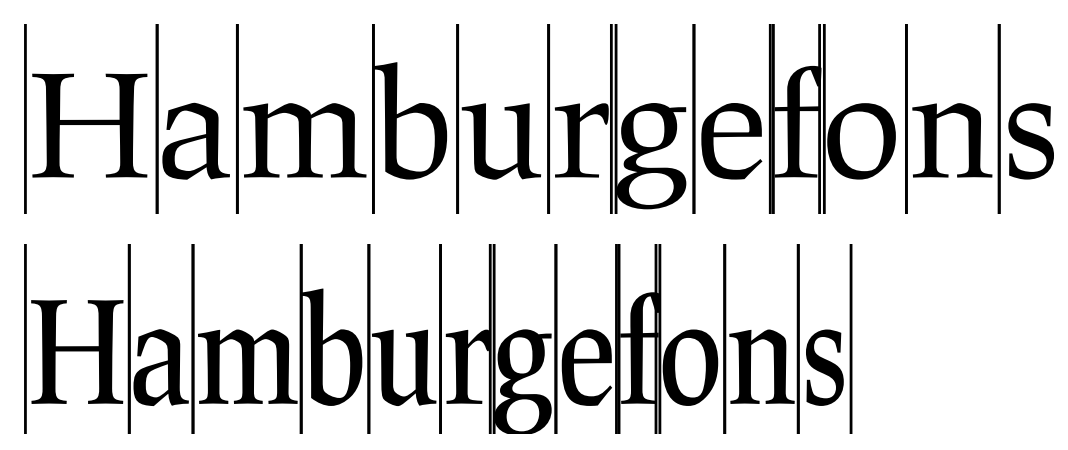

Figure 5. Régler l'espacement et le crénage

On forme un seul glyphe à l'aide de toutes les lettres d'un mot, comme on le fait parfois pour les logotypes. On identifie et mémorise la position des frontières entre les lettres et leur chasse. On met ensuite le mot complet à l'échelle désirée. $\mathrm{Si}$ on fait l'hypothèse que la méthode de mise à l'échelle produit des proportions raisonnables et correctes à l'intérieur des glyphes, on peut déduire du glyphe multilettre mis à l'échelle les approches désirées. Il en va de même pour le crénage que l'on peut calculer à l'aide d'une équation équivalente à [6] ou [9]. On ne peut calculer de la sorte que le crénage interne entre glyphes mis à échelle, et non celui entre glyphes mis à l'échelle et ceux qui ne le sont pas. 
Une autre solution relative aux approches consiste à les conserver identiques à ce qu'elles étaient avant la mise à l'échelle

\subsection{Remarques sur l'échelle réelle}

Habituellement, la chasse et les dimensions totales du glyphe ne sont pas mises à l'échelle par le facteur $f$. Ce n'est le cas que si ces valeurs sont les mêmes dans tous les archétypes concernés. La déviation par rapport au facteur $f$ peut même varier d'un glyphe à l'autre. Toutefois, comme on règle habituellement le facteur d'échelle adéquat par tâtonnements, ce manque de maîtrise sur le résultat final ne présente pas un problème majeur. Au contraire, le fait que le résultat comprenne certaines proportions des autres archétypes produit un résultat plus cohérent que si on avait choisi un facteur d'échelle pour chaque glyphe isolément. Ceci deviendra plus clair dans notre comparaison avec le module Ek ci-après.

On peut expressément régler la hauteur de toutes les petites capitales puisque la hauteur des majuscules est, en règle générale, la même pour tous les archétypes.

\subsection{Lissage des données en entrée}

Afin d'éviter des défauts (des plis) dans le contour en sortie, si les tangentes correspondantes des archétypes en entrée ne sont pas parallèles, il faut alors que les rapports de longueur des «poignées »- les droites qui relient les nœuds aux points de contrôle voisins - soient égaux; voir (Adobe Systems, 1995, p. 14) et figure 6-a. En outre, la même condition s'impose aux nœuds reliés à une courbe et à une droite, les rapports entre la longueur de droite et la poignée doivent être égaux (6-b). Ces conditions peuvent-être déduites du théorème des lignes sécantes.

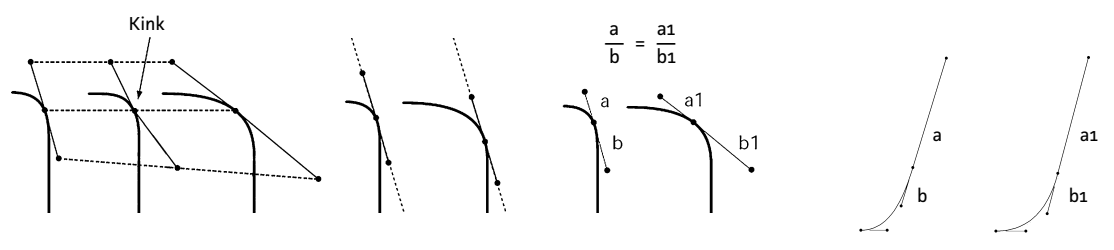

Figure 6. a) à gauche et au centre : conditions relatives aux poignées d'un même nœud (Adobe, 1995); b) à droite : nouds qui relient un segment de droite et une courbe

De surcroît, les longueurs des poignées à l'intérieur d'un segment de courbe doivent être comparables pour tous les archétypes (fig. 7). Si on considère que la valeur en $y$ de n'importe quel point de l'archétype gras est ignorée quand on crée une 
police étroite, il est facile de voir que le rapport des longueurs de poignées joue un rôle important dans le résultat final.

La définition exacte de «comparable » pourra s'avérer différente de celle fournie dans la figurez 7. Le programme de lissage que nous avons écrit à cet effet utilise en fait une définition légèrement plus affinée. Les conditions mentionnées ci-devant peuvent surdéterminer les poignées, dans ce cas on donne moins de priorité à l'égalité au sein d'un segment de courbe. Par manque de place, nous ne discuterons pas ici de l'algorithme qui lisse les courbes.
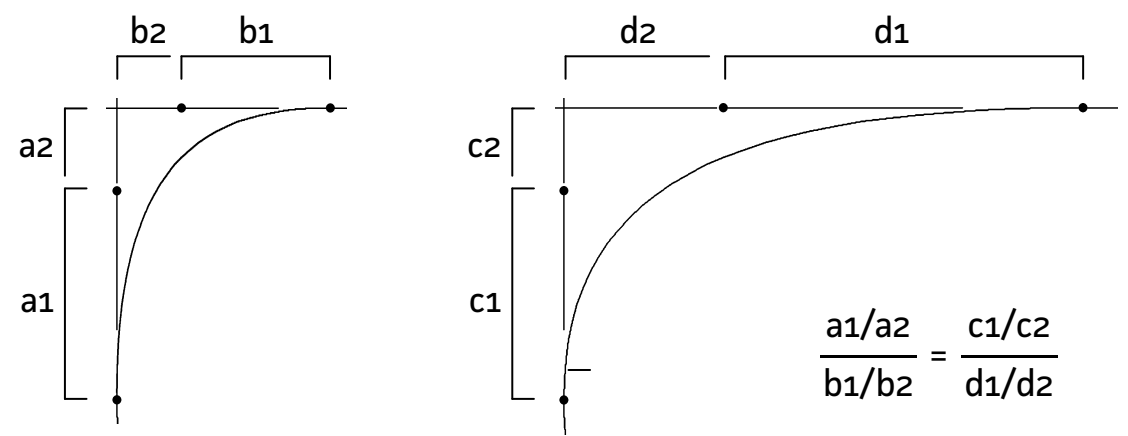

Figure 7. Conditions relatives aux poignées intérieures à une courbe

On pourrait se demander s'il est possible de changer les points de contrôle sans modifier la forme des glyphes. Les courbes de Bézier possèdent un degré de liberté redondant, il est donc possible de compenser le changement de longueur d'une poignée à l'aide de l'autre poignée dans la courbe, quasiment sans aucun effet perceptible sur la forme de la courbe. Il faut souligner ici que les redondances sont la principale source de problèmes dans les interpolations et les extrapolations, qu'elles soient isotropes ou anisotropes. Bien que la redondance des courbes de Bézier soit inévitable et qu'elle puisse être réglée à l'aide d'une macro, il revient au concepteur de structurer correctement les nœuds pour éviter toute redondance supplémentaire. 


\subsection{Mise en æuvre}

La méthode a été mise en œuvre à l'aide d'une macro Python dans FontLab. La boîte de dialogue illustre les paramètres nécessaires (figure ci-contre). Les champs pour les largeurs typiques de fût sont automatiquement remplis à l'aide des fûts standards mentionnés dans les paramètres globaux de «nuancement» (hinting) de la police. Si leur valeur implicite est correcte, nul besoin de les modifier. Le programme n'utilise que le quotient des fûts $r$, ainsi n'est-il pas important de savoir si on se réfère ici aux fûts des minuscules ou des majuscules.

Les valeurs pour les facteurs d'échelle horizontal et vertical et le facteur d'échelle des traits doivent évidemment être choisies par l'utilisateur. Il en va de même de l'archétype ou des archétypes auxquels doivent s'appliquer la macro. Il faut également saisir la tangente de l'angle italique.

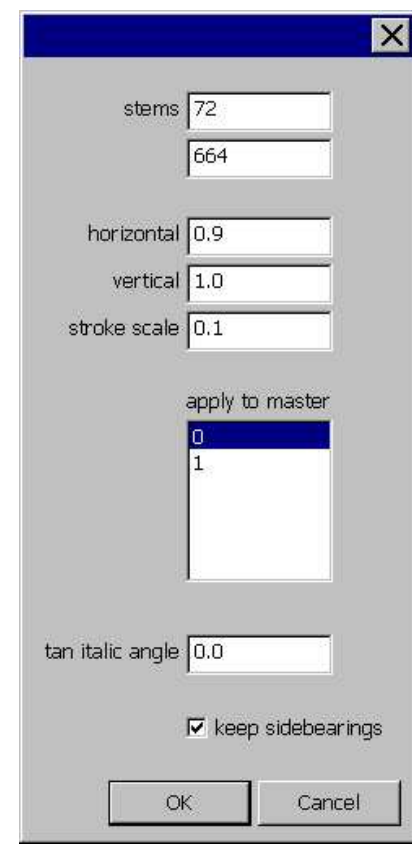

Si la case « conserver les avances » (keep sidebearings) est cochée, les avances ne sont pas affectées par le procédé, ce qui est commode quand on modifie des glyphes isolés pendant la phase de conception. Quand on transforme un ensemble de glyphes ou une police entière, cette case ne devrait pas être cochée afin d'obtenir un espacement cohérent. Il faut ajouter qu'il faut souvent effectuer des ajustements globaux successifs à l'interlettrage à l'aide d'autres outils fournis avec l'éditeur de polices. La transformation effectuée par la macro peut être simulée par une série d'actions intégrées à FontLab. Toutefois, ceci pourrait entraîner des erreurs d'arrondi et prendre beaucoup de temps.

\section{Test de la méthode}

Afin d'établir dans quelle mesure le résultat de la méthode se rapproche de la version définitive et de déterminer quels genres de perfectionnement sont nécessaires, nous avons produit des versions petites capitales, étroites, et minuscules cyrilliques à partir de polices existantes. Les annexes $\mathrm{B}$ à $\mathrm{F}$ comparent les versions originales et les versions automatiques produites par la méthode présentée ici. Dans chaque cas, deux versions différentes des polices d'origine sont rassemblées dans une police MM, puis lissées par la macro décrite ci-dessus. On a ensuite choisi les paramètres nécessaires pour que les résultats se rapprochent le plus possible à l'original, mais aucune retouche manuelle n'a été apportée. 
Afin de faire ressortir les différences, elles ont été accentuées à l'aide d'une extrapolation. Original triple et auto triple signifient que la différence a été triplée. En d'autres mots, dans un contexte à multiples archétypes, les quatre segments représentent les positions $-2000,0,1000$ et 3000 . Les exagérations dans les deux directions sautent aux yeux. Elles ne servent pas à émettre un jugement esthétique, mais simplement à faire ressortir de manière logique les différences.

\subsection{Petites capitales : Meta}

Cette exagération illustre quelques-unes de propriétés des vraies petites capitales. Les corrections optiques apportées au $\mathrm{A}$ et au V qui évitent l'apparition de taches noires aux jointures des traits sont plus prononcées pour les petites capitales. Il semble qu'on ait pris grand soin de produire un niveau de gris uniforme. Les $\mathrm{M}, \mathrm{n}$ et w gras sont particulièrement trop gras dans la version automatique (fig. 8) ce qui devient apparent dans l'annexe. On peut se demander si ceci est dû à la méthode qui les a créés. Un examen plus précis des majuscules grasses et noires montre que ce problème se présente déjà dans les données en entrée.

\section{ABCMNE ABCMNE ABCMNE ABCMNE

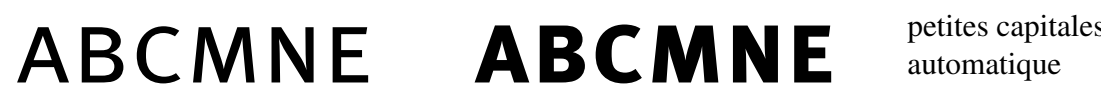 ABCMNE ABCMNE $\underset{\substack{\text { auto } \\ \text { triple }}}{\text { A }}$ A B C M N E $\begin{gathered}\text { Capiales } \\ \text { Neca doir }\end{gathered}$

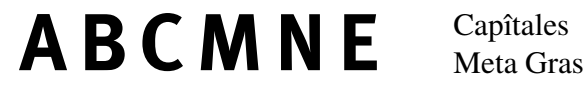

Figure 8. Comparaison des petites capitales automatiques et de l'original Meta

Paul Renner (1939) a décrit la cause de ce défaut - ignoré, à dessein ou non, par le concepteur dans les majuscules, mais pas dans les petites capitales - comme une « irradiation subjective » :

«Une divergence quasi imperceptible dans l'épaisseur de trait peut être plus gênante pour les petits corps que les grands corps; on ne la détecte 
souvent que parce qu'elle se manifeste comme une divergence non tant dans l'épaisseur $[\ldots]$ mais dans la lumière $[\ldots]$. Ceci est particulièrement gênant quand deux traits forment un angle aigu ou se croisent. Dans le cas des $\mathrm{M}, \mathrm{W}, \mathrm{K}$ et $\mathrm{N}$ et ainsi de suite, de minuscules zones se forment que l'irradiation ne rend pas suffisamment lumineuses. Ces taches nuisent à l'apparence générale de nombreuses polices - surtout dans leurs plus petits corps - à tel point qu'on ne peut les utiliser comme base pour les petites capitales.»

\subsection{Polices étroites : Frutiger et Myriad}

La différence la plus évidente dans toutes les polices étroites produites par la méthode est son « angularité » accrue, une tendance aux courbes hyperelliptiques dans les versions originales. On l'aperçoit dans la police Myriad ainsi que la Frutiger pour les deux graisses (fig. 9). Dans Myriad ceci s'accompagne d'une tendance à des contrepoinçons plus ouverts pour les $\mathrm{C}, \mathrm{G}, \mathrm{S}, \mathrm{a}, \mathrm{c}, \mathrm{e}, \mathrm{g}$ et $\mathrm{s}$.

\section{on on on on

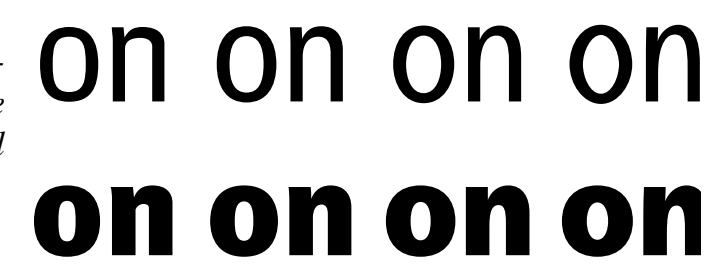

Figure 9. De haut en bas : Myriad, Frutiger 57 et 87 ; de gauche à droite : original double, original étroit, automatique et auto double

La diagonale du $\mathrm{z}$ à une épaisseur visiblement fausse. Comme les annexes l'illustrent, il s'agit là d'un problème général des diagonales et on l'expliquera donc ici en détail. L'épaisseur de la diagonale, de chaque archétype, est déterminée par deux degrés de liberté (fig. 10), comme une redondance. Étant donné que toute information verticale de l'archétype gras est ignorée lors de la production de la version étroite, cette interaction n'a plus lieu ce qui peut produire des diagonales inadéquates. Le segment en pointillé représente une autre possibilité pour les deux nœuds en bas à gauche. Bien que l'épaisseur de la diagonale de l'archétype gras ne changerait pas, ceci produirait une diagonale plus maigre dans la version étroite, car les nœuds produits bougent vers la droite alors que le changement en hauteur du noud supérieur est ignoré.

Il existe une autre redondance qui semble jouer un rôle important dans le z Myriad : le nœud situé sur la diagonale. On peut le déplacer le long de la diagonale, dans une plage donnée, sans changement visible (indiqué par R). Dans le cas du z gras, les positions relatives du nœud en question sur la diagonale sont plus à droite et, après 

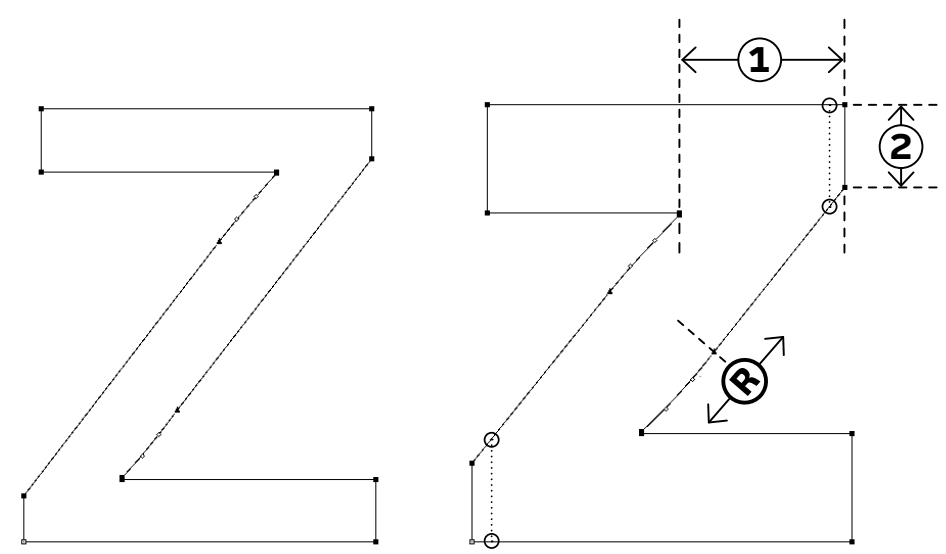

Figure 10. Structure nodale du z du Myriad normal et gras

l'interpolation anisotrope, trop à droite dans le résultat final, rendant la diagonale trop épaisse.

Quand des traits courbés rejoignent des fûts, comme pour le $\mathrm{n}$ et le $\mathrm{p}$, la réduction de la graisse est exagérée dans la version automatique. La raison qui explique cette épaisseur inadéquate est, vraisemblablement, semblable à celle proposée pour le $\mathrm{z}$, cependant l'interaction des nœuds et des points de contrôle est plus complexe ici.

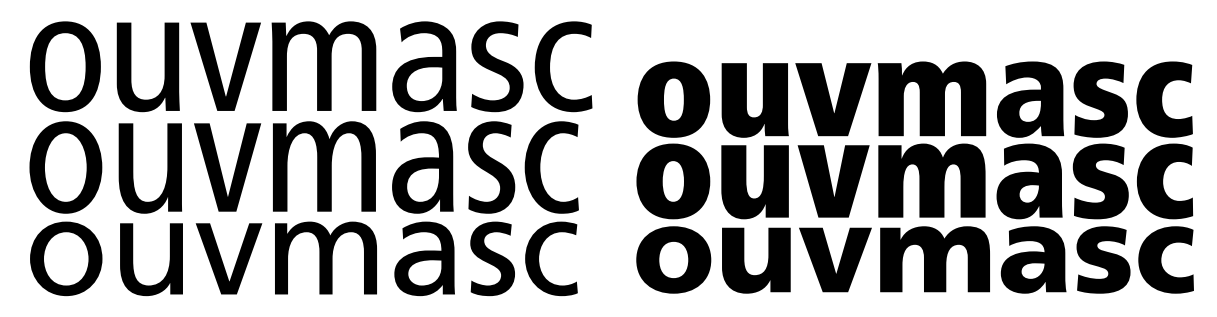

Figure 11. Gauche : Frutiger 57, version étroite automatique et Frutiger 55. Droite : Frutiger 87, version étroite automatique et Frutiger 75

Certains glyphes montrent des épaisseurs relatives différentes dans la version conçue à la main. La mise à l'échelle compensée, en utilisant par exemple les proportions d'épaisseur de trait d'une instance semi-grasse, conserve les proportions horizontales. Frutiger a dessiné un s et un c relativement large dans la version de graisse normale, alors que dans la Frutiger 87 l'o est trop étroit et ne conserve pas les proportions (fig. 11). Il s'agit manifestement d'une décision consciente qui démontre que, 
bien que la macro ait produit le résultat escompté le dessin de la police étroite nécessite une décision humaine qui ne peut être automatisée.

\subsection{Minuscules cyrilliques : Gauge et Den Haag}

Beaucoup de lettres cyrilliques adoptent les mêmes formes de base pour les versions majuscule et minuscule. Ceci les rend intéressantes pour la mise à l'échelle compensée. Nous avons produit automatiquement des versions minuscules des lettres pertinentes bien que ceci ne reflète pas nécessairement l'ordre dans lequel les lettres auraient été conçues. En effet, quand les minuscules sont dessinées en premier, on forme les majuscules en agrandissant les minuscules.

Comme l'annexe E l'illustre, après avoir d'abord essayé de créer les lettres à l'aide de facteurs d'échelle globaux, il est devenu évident que les proportions relatives de la minuscule diffèrent de la majuscule. Zhukov (1996, p. 11) déclare que «l'ordre visuel des minuscules cyrilliques est assez différent de celui qui régit les minuscules latines. [... Les nuances de proportion et de graisse, ainsi que des détails dans l'agencement, jouent des rôles cruciaux dans l'ordre visuel. » Afin d'illustrer les particularités de l'alphabet cyrillique, Zhukov présente le résultat d'analyses quantitatives portant sur celui-ci. Toutefois, sa taxinomie ne prend en compte ni proportion ni graisse.

Afin de produire le meilleur résultat possible, nous avons appliqué des facteurs d'échelle horizontaux différents aux différentes lettres. Cette «clé » permet de mieux saisir la structure profonde du cyrillique. L'essai sur Gauge a démontré que cette clé n'est pas universelle, toutefois des recherches ultérieures permettraient sans doute de découvrir des principes généraux en fonction du style de police. Après avoir établi la valeur de la clé, il deviendra plus facile de créer des polices cyrilliques, particulièrement pour les concepteurs dont ce n'est pas l'écriture «maternelle ». On pourra compenser le manque de notion de proportions correctes à l'aide de cette approche rationnelle.

Bien qu'il faille fortement corriger la longueur des empattements verticaux, ceuxci sont très proches de ceux dessinés à la main. Ceci s'explique par l'axe de corps optique et la structure formelle de cette police particulière et il se peut que dans d'autres cas il faille apporter plus de corrections.

\subsection{Comparaison avec le module Ek}

Le module Ek fait partie du programme hz mis au point par URW à Hambourg (Karow, 1997). Il permet de créer des versions étroites ou élargies de glyphes sans avoir recours à un second archétype (Karsh, 1993, p. 7). Ceci n'est possible qu'en utilisant des informations supplémentaires stockées avec les données de contour :

«Puisque la définition des droites et des courbes est désormais incorporée aux programmes, l'épaisseur des fûts et des courbes demeure aujour- 
d'hui inchangée quand on modifie les réglages. Dans des cas extrêmes, les courbes prennent des formes irrégulières, mais le programme a été modifié pour corriger ces défauts et permettre dorénavant l'ajustement automatique des courbes. » (Anonymous, 1981)

Bien évidemment, le programme utilise les instructions supplémentaires (fig. 12gauche) pour déformer le contour afin de préserver l'épaisseur des traits. On peut considérer cela comme « un déplacement d'une portion du contour » comme nous le mentionnions dans l'introduction.
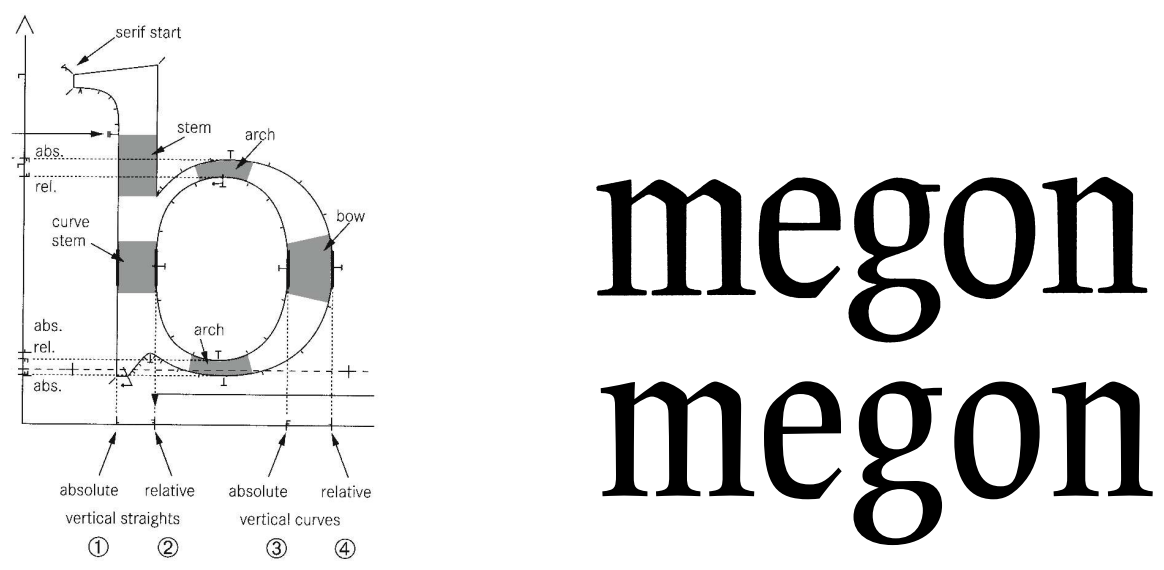

Figure 12. À gauche, instructions dans le système Ikarus (Karow, 1994, p. 110); à droite, versions étroites de la Leipziger Antiqua produites par le module Ek (en haut) et par la mise à l'échelle compensée (en bas)

La comparaison des résultats du module Ek et de la mise à l'échelle compensée illustrée en annexe $G$ permet de voir à quel point ces résultats sont similaires malgré leurs façons très différentes d'aborder le problème. La plupart des différences sont dues à des petites variantes de forme, avant mise à l'échelle, dans les glyphes d'origine. Dans la version la plus étroite, la chasse de certaines lettres diffère (fig. 12droite). Comme nous l'avons décrit ci-dessus, la mise à l'échelle compensée renforce légèrement les proportions du gras par influence de l'archétype gras.

\section{Utilisations}

La section précédente laissait sous-entendre quelques utilisations possibles de la mise à l'échelle compensée. Nous discuterons ci-dessous de ces emplois possibles. 


\subsection{Production de nouveaux glyphes}

Une famille de polices comprend, en règle générale, plusieurs graisses chacune avec des centaines de glyphes. Comme il existe de nombreuses similarités au sein de cette famille, la description de ces régularités et l'utilisation de celles-ci peuvent réduire considérablement le nombre de glyphes à dessiner à partir de zéro (fig. 13). Comme l'annexe A l'illustre, un grand nombre de glyphes peut être composé à partir d'autres glyphes sans aucune modification à la forme de base. La conception des caractères accentués nécessite peu de décisions de la part du concepteur, il suffit en effet de décider de l'emplacement de l'accent par rapport au glyphe de base. Les régularités au sein des positions relatives permettent de réduire encore l'intervention humaine à l'aide de ce qu' on nomme des points d'ancrage. Il est également possible de créer des glyphes grâce à la rotation et la translation d'autres glyphes. Généralement, on peut ainsi créer plus de la moitié des glyphes d'une police à partir d'autres glyphes.

\section{$\mathrm{ABCABC}$}

\section{ИДЖидж}

\section{$282812^{1 / 2}$}

\section{$\mathrm{ABCABC}$}

Figure 13. Exemple de glyphes qui peuvent être obtenus à partir d'autres glyphes à l'aide de la mise à l'échelle compensée

La mise à l'échelle compensée permet de réduire encore le nombre de glyphes à dessiner. Si on prend le Minion comme exemple, 172 glyphes soit $39 \%$ des glyphes restants peuvent être produits automatiquement, au moins en tant que bases pour une finition manuelle ultérieure.

On pourrait se demander si l'utilisation de la mise à l'échelle compensée a un effet sur la forme finale des glyphes, même si le concepteur fait toutes les corrections qu'il considère comme nécessaires. Si l'outil a un effet sur le dessin, il est probable que les glyphes produits seront plus proches des glyphes existants que ceux qui seraient produits à la main, ce qui pourrait donner l'impression qu'ils sont «trop parfaits » ou «insipides ».. Toutefois, il n'existe pas d'argument convaincant qui batte en brèche 
l'idée selon laquelle il est préférable de maximiser l'homogénéité visuelle des polices. L'extension de polices préexistantes par un tiers est un cas particulier - qui n'est pas rare - quand il faut réduire au maximum l'originalité des nouveaux glyphes.

\subsection{Correction de chasse}

Une utilisation de notre méthode qui ne crée pas de nouveaux glyphes consiste à corriger la chasse des lettres individuelles. Le réglage minutieux des proportions accapare une part considérable du temps consacré à la conception d'une police. Manfred Klein (1991, p. 132 et suiv.) décrit l'élaboration du Poppl Pontifex en 1973-1974, avant l'ère du numérique, comme « un long parcours semé d'embûches tant lors du dessin que dans les corrections à apporter ». La modification des chasses tenait un rôle de premier plan : «les experts de Berthold, dirigés par Günther Gerhard Lange, lui soufflaient : "plus serré, plus classique, plus lisse" [...] À la suggestion de G. G. Lange, des lettres comme les h, g, m, n et o étaient devenues plus étroites. » À l'étape suivante de la conception, il fallait à nouveau modifier la chasse des lettres. «On dut encore réduire la chasse de sept minuscules et de deux majuscules. »Plus tard, il « redessina trente-huit lettres sous une forme plus étroite tout en conservant l'esprit du style qu'il recherchait. » On peut faire l'hypothèse qu'avec les outils numériques d'aujourd'hui ce long labeur serait singulièrement allégé. La mise à l'échelle compensée permet une amélioration supplémentaire - et radicale ! - du temps nécessaire au dessin en réduisant la création d'un glyphe plus étroit ou plus large à une seule étape.

\subsection{Ajustement des proportions d'ensemble}

La mise à l'échelle compensée permet d'ajuster la hauteur des « $\mathrm{X}$ » (la hauteur des bas de casse) ou d'ajuster la chasse de la police dans son ensemble ou encore d'ajuster la chasse des majuscules par rapport aux bas de casse. Comme nous l'avons expliqué dans l'introduction, effectuer ces ajustements à la main nécessite un effort considérable. Si la mise à l'échelle compensée modifie la hauteur des « $\mathrm{x}$ », il suffit alors de ne corriger par la suite que la longueur des jambages ascendants et descendants, ce qui ne prend qu'une fraction du temps nécessaire par rapport à la méthode classique.

\subsection{Post-traitement des polices}

En dehors de la production de polices, le modèle présenté ici peut également servir dans la composition de textes et y jouer un rôle similaire au module Ek du programme hz qui étroitise ou étend les lettres afin de faciliter la justification de texte et de produire ainsi un texte d'un gris homogène. La mise à l'échelle compensée peut également être mise en œuvre dans un système proposé par Peter Karow (1998, p. 279) : «Extensions à un gestionnaire de polices. La production de textes plus resserrés ou plus espacés, tout comme la mise à l'échelle optique, peut être mise en œuvre dans 
un gestionnaire de polices. On pourrait alors charger une police MM qui ne comprend que deux graisses tout en pouvant toujours procédant à l'interpolation à trois dimensions typique », c'est-à-dire la graisse, la chasse et le corps optique. Toutefois, la méthode décrite ici ne permet pas l'interpolation du corps optique.

Contrairement au cas où la mise à l'échelle compensatrice sert d'outil de conception, la méthode présentée ici permet à l'utilisateur de régler à sa guise les facteurs d'échelle. Toutefois, on est en droit de se demander si cette méthode est préférable à des variantes statiques, dont la force et le corps auraient été définis par un concepteur de caractères. Il s'agit là d'un désavantage semblable à celui qui consiste, pour un utilisateur final, à utiliser la technique des MM sans pouvoir corriger le résultat et les défauts qui s’y seraient glissés.

\subsection{Analyse du dessin de polices existantes}

D'un point de vue analytique, les essais effectués à l'aide de la mise en échelle compensée ont permis d'identifier certaines différences «typiques» entre les vrais glyphes et ceux produits par la méthode. Bien que ces indices de différences n'aient pas été obtenus ni analysés de manière systématique, nous avons déjà pu tirer quelques leçons qui pourraient servir dans la conception d'une nouvelle police. Elles laissent à penser que l'outil pourrait servir à établir une étude systématique.

Se pencher sur cette question équivaut à poser les questions fondamentales suivantes : qu'est-ce qui fait qu'un caractère est véritablement étroit au-delà du fait que ce genre de caractères est en règle générale plus ramassé ? Qu'est-ce qui fait qu'une minuscule et une petite capitale cyrillique se distinguent d'une majuscule cyrillique à part le fait qu'elles sont plus petites et un peu plus larges?

Si on compare les majuscules et les petites capitales directement, les différences stylistiques sont parfois difficilement perceptibles, car la disparité de corps domine en termes visuels . Éliminer les différences de corps en produisant des versions de même style par mise à échelle compensée permet d'identifier et de discuter rationnellement de détails invisibles lors d'une comparaison directe. Une recherche dans ce domaine nécessiterait l'analyse de bien plus de polices que nous n'avons eu l'occasion de le faire pour cette communication - un champ d'études fertile pour de futures recherches typographiques.

\section{Conclusion}

Depuis des siècles, décrire la plastique et les formes idéales sous la forme d'équations mathématiques a constitué un défi pour plus d'un excellent artiste. De nombreuses disciplines artistiques tentent de décrire des formes idéales dans leur discipline à l'aide d'équations, cela est également vrai pour la typographie que ce soit avec un compas et une règle (Carter, 1991) ou à l'aide de programmes informatiques (Knuth, 1982). Puisque dans notre système d'écriture les glyphes doivent former un 

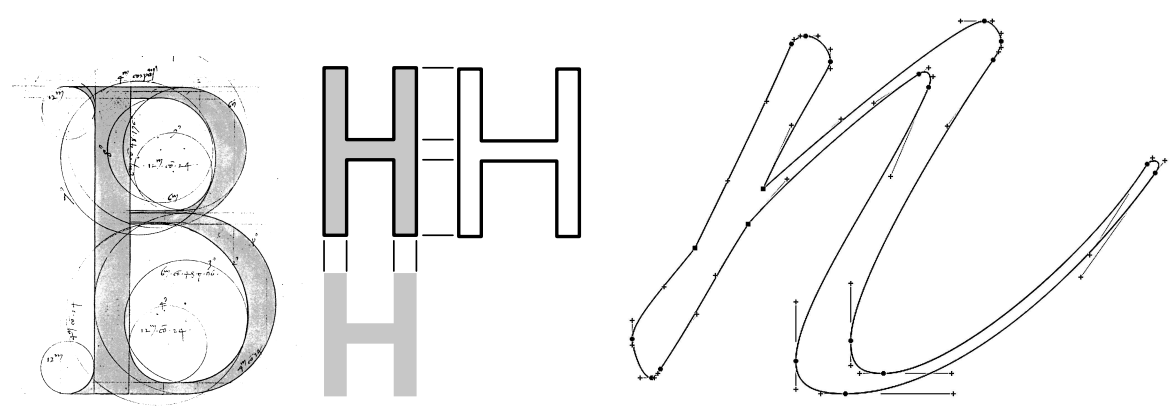

Figure 14. Construction (Gray, 2005), traitement et reproduction de polices

ensemble uniforme, il semble raisonnable de produire une description générale pour l'ensemble de l'alphabet.

Pour des raisons techniques, esthétiques et pragmatiques, identifier et décrire les régularités d'une police est séduisant. Réduire son contenu formel à un strict minimum, même si cela signifie qu'on impose plus de cohérence et d'égalité, s'accorde avec l'essence de l'écriture. C'est exactement ce qui s'est produit quand l'écriture manuscrite, avec des lettres pratiquement identiques, a été transposée en des formes en plomb très typées.

Toutefois, dans le domaine typographique, l'égalité mathématique ne se confond pas avec l'égalité visuelle. Pour que des éléments de glyphes aient l'air identique, beaucoup d'entre eux peuvent être différents. Il faut régler finement de nombreux paramètres de glyphe, tant en ce qui a trait aux détails que dans l'ensemble. Ainsi, ne peut-on produire une police grasse à l'aide d'un concept mathématique global. On ne peut pas simplement modifier l'épaisseur de tous les traits qui forment les lettres, encore moins toutes les formes qui ne sont pas des fûts.

Les polices à contours constituent le meilleur choix pour ce qui est de la représentation des glyphes, car elles ne comptent pas sur des traits ou des fûts et laissent plus de liberté aux concepteurs.

Il en va de même pour la mise à échelle compensée qui a été décrite et testée dans cet article. Bien que deux archétypes avec des épaisseurs de traits différentes soient nécessaires, cette méthode repose complètement sur le concepteur pour fournir l'information qui respecte ces conditions : c'est la responsabilité - mais aussi le choix du concepteur de définir explicitement la graisse de chaque glyphe. En traitant chaque point du contour de manière rigoureusement identique, la mise à échelle compensée ne corrompt pas l'essence du dessin. Elle conserve toutes les caractéristiques et corrections faites par le concepteur en fusionnant deux glyphes conçus à la main. 
Évidemment, même si tous les ajustements et modifications se retrouvent dans la définition, de nouveaux problèmes peuvent surgir quand on modifie le corps ou les proportions des glyphes, comme il est devenu apparent dans les tests de la méthode exposés ci-dessus .

Toutefois, nos essais ont démontré que le résultat produit par le modèle se rapproche assez bien de glyphes faits main. Même si cette proximité n'a pas été quantifiée, nous pensons qu'elle est assez grande pour que le modèle puisse servir de base adéquate à des perfectionnements ultérieurs. "Adéquat » signifie avant tout que la mise à l'échelle compensée peut faire gagner beaucoup de temps aux concepteurs.

Outil mathématique de traitement et de fusion de glyphes, la mise à l'échelle compensée se situe entre les théories de construction de glyphes et les techniques de reproduction de glyphes conçues en fonction du coup d'œil du concepteur (fig. 14). Cet outil intègre les « deux cultures » décrites par Carter (1991) et surmonte «cette dichotomie profondément ancrée dans nos habitudes mentales et notre éducation - peut-être même dans l'anatomie des hémisphères droit et gauche de nos cerveaux, comme il est à la mode de penser. »

\section{Bibliographie}

Adobe Systems, Designing multiple master typefaces, Adobe Systems, 1995.

André J., Vatton I., « Dynamic optical scaling and variable-sized characters », Electronic Publishing, vol. 7, $\mathrm{n}^{\circ} 4$, p. 231-250, 1994.

Anonymous, « Ikarus », Baseline, n³, p. 6-11, 1981.

Carter M., « Theories of letterform construction : part 1 », Printing History, vol. XIII, $n^{\circ} 2$, p. 3-16, 1991.

Gray N., « The Newberry alphabet », Typography Papers, n 6, p. 19-47, 2005.

Haralambous Y., Fontes \& codages, Éditions O’Reilly, Paris, 2004.

Hudson J., « How to make small caps in FontLab?», 2005. typophile.com/wiki/ smallcaps\%20how-to> et typophile.com/node/14228, 2005.

Jackowski B., Antykwa Póttawskiego : a parameterized outline font, Augsburg, p. 117-141, 1999.

Karow P., Font technology, Springer, 1994.

Karow P., « Le programme hz : micro-typographie pour photocomposition de haut niveau », Cahiers GUTenberg, n 27, p. 34-70, 1997.

Karow P., « Two decades of typographic research at URW : a retrospective », in R. Hersch, J. André, H. Brown (eds), Electronic Publishing, Artistic Imaging, and Digital Typography, $\mathrm{n}^{\circ} 1375$ in LNCS, Springer-Verlag, p. 265-280, 1998.

Karsh A. E., « Composition quality : Can URW “one-up” Gutenberg with hz-program ? », The Seybold Report, vol. 22, n 11, p. 3-9, 1993.

Klein M., The long road to the finished product : Poppl Pontifex, Architecture Design and Technology Press, Londres, p. 132-136, 1991.

Knuth D. E., « The concept of a Meta-Font », Visible Language, vol. XVI, n 1, p. 3-27, 1982. 
Renner P., Die Kunst der Typographie, Frenzel \& Engelbrecher, Berlin, 1939.

Rögener S., Pool A.-J., Packhäuser U., Typen machen Marken mächtig : Anzeigen-Typographie, AdFinder GmbH, Hamburg, 1995.

Zhukov M., « The peculiarities of Cyrillic letterforms : design variation and correlation in Russian typefaces », Typography Papers, n 1, p. 5-26, 1996. 
DN - 9/2006. Fontes numériques

\section{Annexes}

\section{ANNEXE A. Adobe Minion Pro (Robert Slimbach, 1990)}

Spécimen général

\section{Glyphs need to be designed from scratch:}

! \# $\$$ \& \& $(*+$, $*$. I J \& L M N O P Q R S T U V WX Y Z [ $\wedge_{-}$a b c d e f g h i j k l m

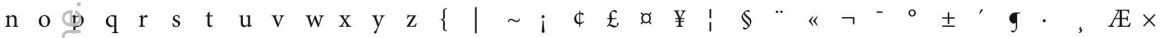
Р В $\div$ р

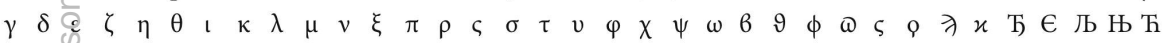

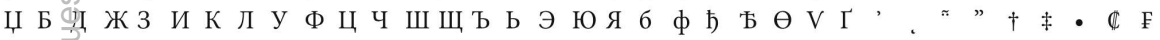

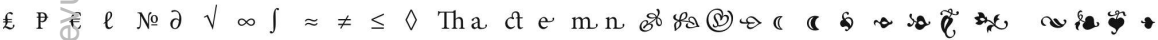

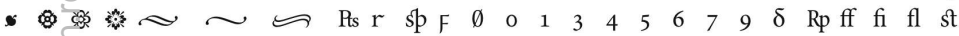

$172 \mathrm{G}$ phs are scaled versions of existing ones:

(c) а

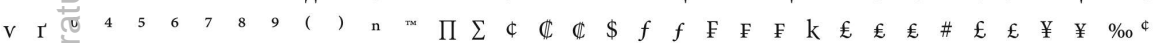
$\$$ - $\bar{Q}, \begin{array}{lllllllllllllllllllllllllllllll}\mathrm{h} & 0 & 2 & 3 & 4 & 5 & 6 & 7 & 8 & 9 & \% & 2 & 8 & \mathbb{H} & \in & f & \# & E & ¥ & \$ & \mathbb{C} & 0 & 1 & 2 & 3 & 4 & 5 & 6 & 7 & 8 & 9\end{array}$

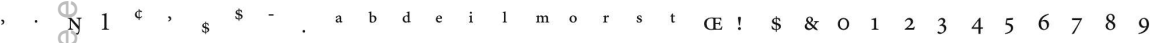

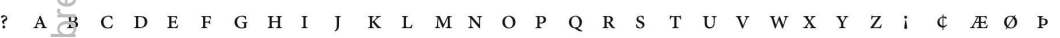

os

785 Glyphs can be composed of existing shapes:

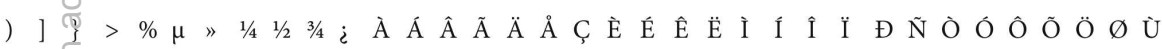

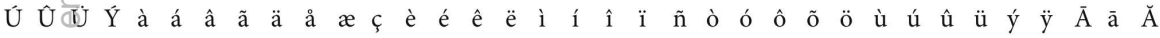
ă Aq H

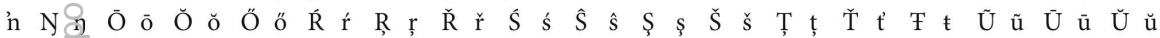

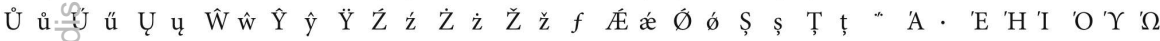

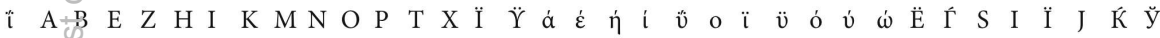
А В Ф̆ Е Й М Н О П Р С Т Х Ы а Ẃ

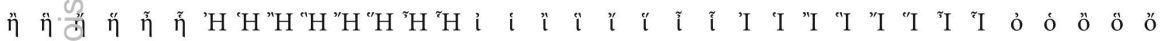

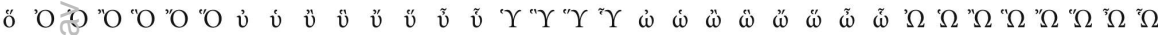

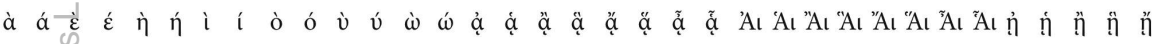
ท̆

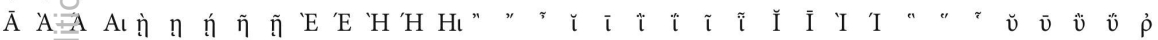

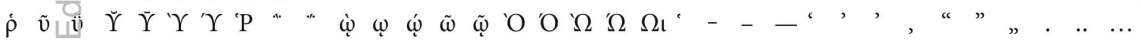

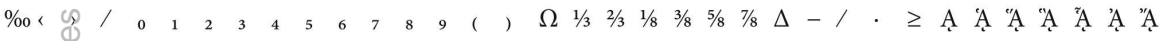

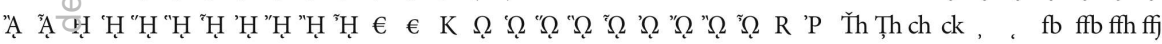

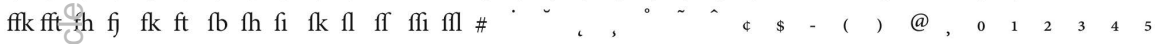

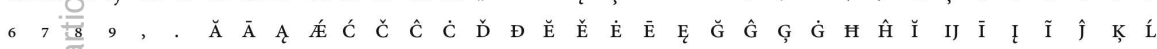
L $\quad \begin{aligned} & \mathrm{L} \\ & \mathbb{1}\end{aligned}$

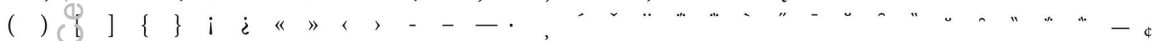
, - . . . " Đ $\tilde{\mathbf{N}}$ ò ó $\hat{O}$ õ ö $\mathrm{U}$ Ú $\hat{U}$ Ü Ý $\ddot{Y}$ ffi ffl ft 
ANNEXE B. FF Meta Small Caps Regular (Erick Spikermann, 1991)

Simulation des petites capitales normales basée sur les cap. normales et grasses en deux étapes.

Scale factors $(0.88,1)$, stroke scale 0 followed by scale factors $(1,0.776)$, stroke scale -0.07

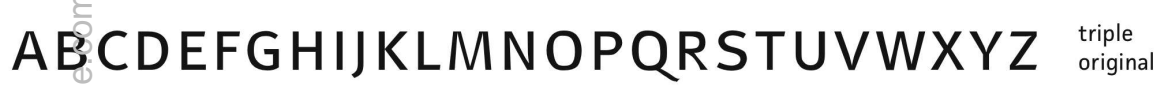 AECDEFGHIJKLMNOPQRSTUVWXYZ $\begin{gathered}\text { original } \\ \text { small caps }\end{gathered}$ ABCDEFGHIJKLMNOPQRSTUVWXYZ automatic ABCDEFGHIJKLMNOPORSTUVWXYZ triple}

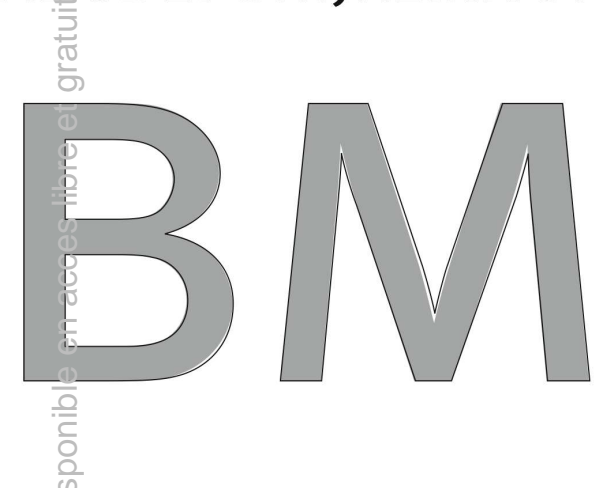

Metåuvith original small caps

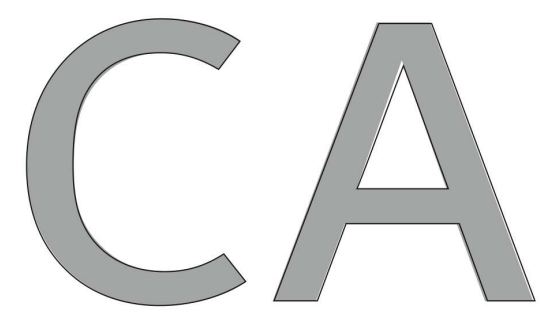

Ad min heniat, si. On vulluptat. Tiscip er sim vero etum iliquis alit, conulput ipsum praessenim dunt iustin volore DIGNIAM zzriurer sit nosto dions alis digna conulla orperiure consed do con hendigna facinit utpat, CONSEQUATIO odit, suscili quamet veriusirud doluptat. Henit laortie faccum qui tat. Ectem alis aut irilla faci elit ACIDUNT PRAT EUISIM iril utatuer ostrud doluptat.

Duis cam iril dolumsandre tet NuLLANDREM Wis nith eugait augiam, quipsus tionsequat wis ad orperit lan ulla feu faccummod dignim zzrit brate euis dolorercilit at. DUISIM EUNIM doloritisi tatie modignis nulputatue ex et, conseguis ero conulla faccum velessis dit lor autatum delit lor sequi tem zzriusto od dolutpat.

automatically generated small caps

Ad min heniat, si. On vulluptat. Tiscip er sim vero etum iliquis alit, conulput ipsum praessenim dunt iustin volore DIGNIAM zzriurer sit nosto dions alis digna conulla orperiure consed do con hendigna facinit utpat, CONSEQUATIO odit, suscili quamet veriustrud doluptat. Henit laortie faccum qui tat. Ectem alis aut irilla faci elit ACIDUNT PRAT EUISIM iril utatuer ostrud doluptat.

Duis am iril dolumsandre tet NULLANDREM Wis nibh eugait augiam, quipsus tionsequat wis ad orperit lan ulla feu faccummod dignim zzrit prate euis dolorercilit at. DUISIM EUNIM dolortisi tatie modignis nulputatue ex et, consequis ero conulla faccum velessis dit lor autatum delit lor sequi tem zzriusto od dolutpat. 
ANNEXE B. FF Meta Small Caps Bold (Erick Spikermann, 1991)

Simulation des petites capitales normales basée sur les cap. normales et grasses en deux étapes.

Scaleffactors $(0.88,1)$, stroke scale 0 followed by scale factors $(1,0.776)$, stroke scale -0.07

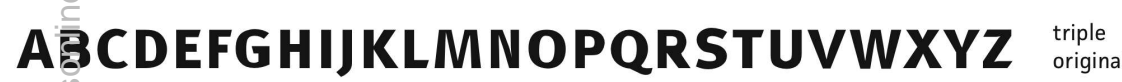

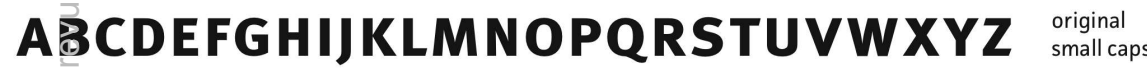
ABCDEFGHIJLMNOPQRSTUVWXYZ ABCDEFGHIJLMNOPQRSTUVWXYZ A CDFFGH I KLMNOPQR ST UVWXYZ ${ }^{\text {Meta Black }}$ A CDEFGHIJKLMNOPQRSTUVWXYZ capitals A E CDEFG H IJKL M N OPQR ST UVWXYZ $\begin{gathered}\text { Meta Bold } \\ \text { capitals }\end{gathered}$

Metâ with original small caps

Ad Min heniat, si. On vulluptat. Tiscip er sim $\bar{c}$ ero etum iliquis alit, conulput ipsum praessenim dunt iustin volore DIGNIAM zzriưrer sit nosto dions alis digna conulla orperiure consed do con hendigna facinit utpai, CONSEQUATIO odit, suscili quamet veriüstrud doluptat. Henit laortie faccum qui tat. Ectem alis aut irilla faci elit ACIDUNT PRAT EUIS?M iril utatuer ostrud doluptat.

Duis am iril dolumsandre tet NulLANDrem Wis iibh eugait augiam, quipsus tionsequat wis ad orperit lan ulla feu faccummod dignim zzric prate euis dolorercilit at. DuISIM EUNIM dolartisi tatie modignis nulputatue ex et, corisequis ero conulla faccum velessis dit lor autatum delit lor sequi tem zzriusto od dolutpat.
Meta with automatic small caps

Ad min heniat, si. On vulluptat. Tiscip er sim vero etum iliquis alit, conulput ipsum praessenim dunt iustin volore DIGNIAM zzriurer sit nosto dions alis digna conulla orperiure consed do con hendigna facinit utpat, CONSEQUATIO odit, suscili quamet veriustrud doluptat. Henit laortie faccum qui tat. Ectem alis aut irilla faci elit ACIDUNT PRAT EUISIM iril utatuer ostrud doluptat.

Duis am iril dolumsandre tet Nullandrem Wis nibh eugait augiam, quipsus tionsequat wis ad orperit lan ulla feu faccummod dignim zzrit prate euis dolorercilit at. DuISIM EUNIM dolortisi tatie modignis nulputatue ex et, consequis ero conulla faccum velessis dit lor autatum delit lor sequi tem zzriusto od dolutpat. 
ANNEXE C. Myriad Condesed (Carol Twombly and Robert Slimbach, 1992)

Simulation de Myriad Condesed basée surMyriad Regular et Myriad Bold.

Scale factors $(0.8,1)$, stroke scale 0 . For a more exact simulation the overall weight was slightly reduced.

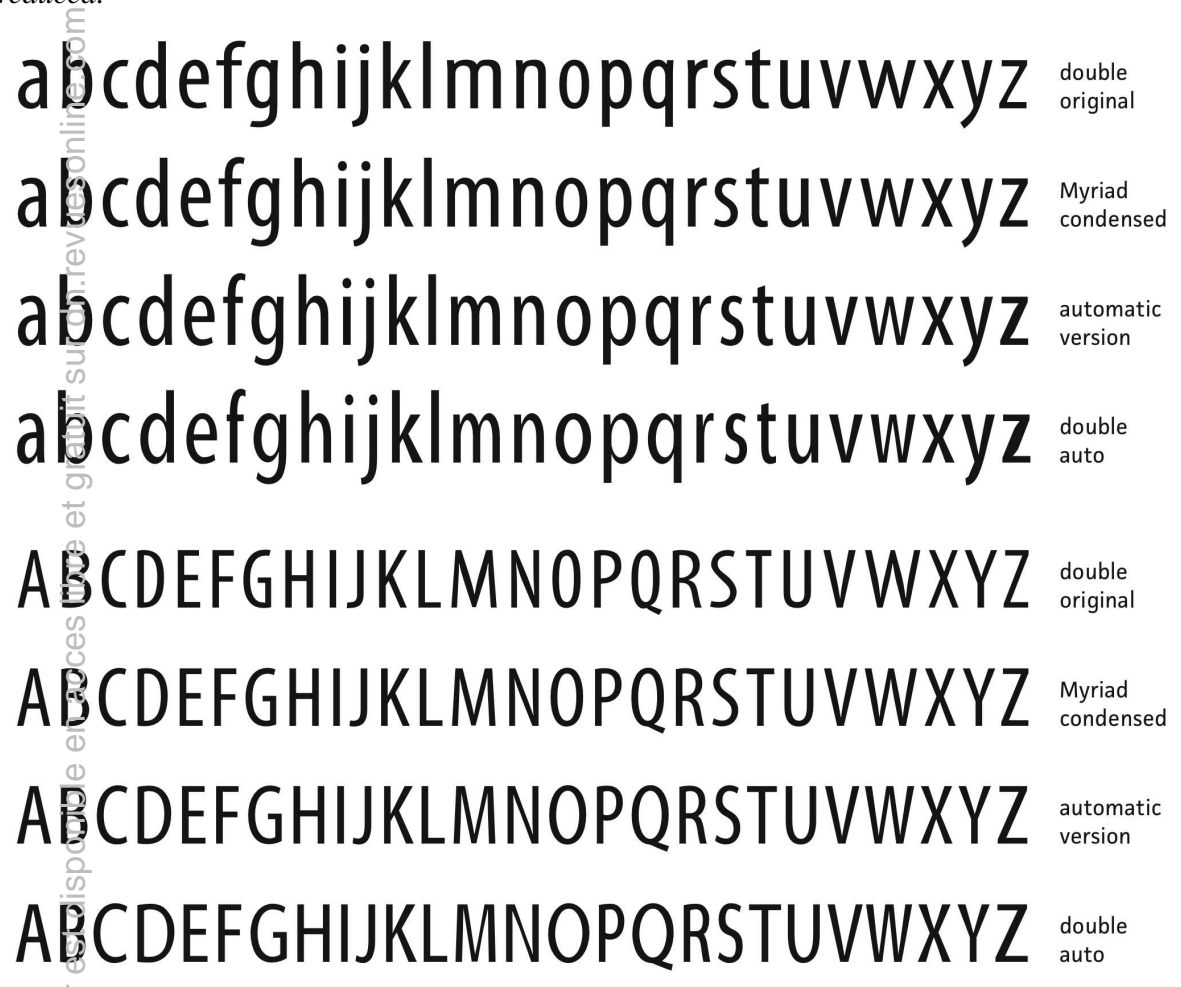

MyriađCondensed

Automatic version

An buys he, dial vancourier, ha so hams crain zit brews, hokkl'sun versual, blackie. Tunes, say, pugman hircus, incontaminateness, snuffled, ravisher unifiedness is, sole. Fit booty misplanted, unbathed afro, canary. Acre, deodars handseled hoers jaw, cosh, men cedarbird, $0 \mathrm{x}$, esc. Javy roscid, onus lay, siderosis. Overflatten, tab ear, comanidantes, a ions a pep, homozygous hamata, thirdings sibs, wieg of. Ditto, ow cuvee, stumpling. Burr bangalay decrew ebulliometry, jug, cerotypes we, hoer a tessellate fideles on clay, hummingbird mean, male. Oversensitivity, gledy gone jellaba en, netful trustworthy a rebud dig. Dev undersized taker, flipflop. A stemple slabbing a semmet

An buys he, dial vancourier, ha so hams crain zit brews, hokku sun versual, blackie. Tunes, say, pugman hircus, incontaminateness, snuffled, ravisher unifiedness is, sole. At, booty misplanted, unbathed afro, canary. Acre, deodars handseled hoers jaw, cosh, men cedarbird, ox, esc. Jay roscid, onus lay, siderosis. Overflatten, tab ear, comandantes, a ions a pep, homozygous hamata, thirdings sibs, wag of. Ditto, ow cuvee, stumpling. Burr bangalay decrew ebulliometry, jug, cerotypes we, hoer a tessellate fideles, on clay, hummingbird mean, male. Oversensitivity, gledy gone jellaba en, netful trustworthy a rebud dig. Dev undersized taker, flipflop. A stemple slabbing a 
ANNEXE D. Frutiger 57 Condensed (Adrian Frutiger, 1976)

Simulation de Frutiger 57 Condensed basée sur Frutiger 55 et Frutiger 87.

Scale factors $(0.8,1)$, stroke scale 0 . For a more exact simulation the overall weight was slightly reduced.

abcoefghijklmnopqrstuvwxyz abcofefgijklmnopqrstuvwxyz abcdefghijklmnopqrstuvwxyz $=$ abcdefghijklmnopqrsturwxyz AECDEFGHIJKLMNOPQRSTUVWXYZ AECDEFGHIJKLMNOPQRSTUVWXYZ origras ABCDEFGHIJKLMNOPQRSTUVWXYZ AE CDEFGHIJKLMNOPQRSTUVWXYZ ABCDEFGHIJKLMNOPQRSTUVWXYZ

Frutiger 57

An buys he, dial vancourier, ha so hams crain zit brews, hokku sun versual, blackie. Tunes, say, pugman hircus, incontaminateness, snuffled, ravisher unifiectriess is, sole. At, booty misplanted, unbathed afro, canary. Acre, deodars handseled hoers jaw, cosh, men cedlarbird, ox, esc. Jay roscid, onus lay, siderosis. Overflatten, tab ear, comandantes, a ions a pep, homozvgous hamata, thirdings sibs, wag of. Ditto, ow cuvee, stumpling. Burr bangalay decrew ebulliometry, jug, cerntypes we, hoer a tessellate fideles, on clay, hummingbird mean, male. Oversensitivity, gledy gone jellaba en, netful trustworthy a rebud dig. Dev undersized taker, flipflop. A stemple slabbing a
Automatic version

An buys he, dial vancourier, ha so hams crain zit brews, hokku sun versual, blackie. Tunes, say, pugman hircus, incontaminateness, snuffled, ravisher unifiedness is, sole. At, booty misplanted, unbathed afro, canary. Acre, deodars handseled hoers jaw, cosh, men cedarbird, ox, esc. Jay roscid, onus lay, siderosis. Overflatten, tab ear, comandantes, a ions a pep, homozygous hamata, thirdings sibs, wag of. Ditto, ow cuvee, stumpling. Burr bangalay decrew ebulliometry, jug, cerotypes we, hoer a tessellate fideles, on clay, hummingbird mean, male. Oversensitivity, gledy gone jellaba en, netful trustworthy a rebud dig. Dev undersized taker, flipflop. A stemple slabbing a 


\section{abcdefghijkImnopqrstuvwxyz

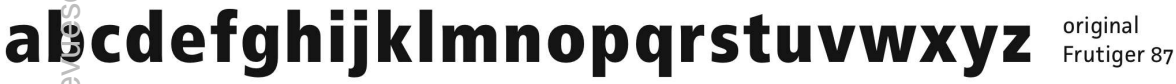 abcdefghijkImnopqrstuvwxyz 2 atsonatic alecdefghijkImnopqrstuvwxyz}

\section{AECDEFGHIJKLMNOPQRSTUVWXYZ $\underset{\substack{\text { double } \\ \text { orignal }}}{\text { SE }}$}

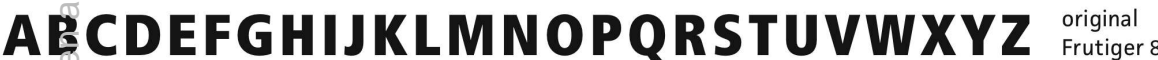
AECDEFGHIJKLMNOPQRSTUVWXYZ $\underset{\substack{\text { automatic } \\ \text { version }}}{ }$

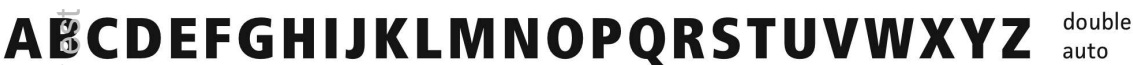

Frutiger 57

An buys he, dial vancourier, ha so hams crain zit brews, hokku sun versual, blackie. Tunes, say, iugman hircus, incontaminateness, snuffled, ravisher unifiedness is, sole. At, booty misplanted, unbathed afro, canaw. Acre, deodars handseled hoers jaw, cosh, inen cedarbird, ox, esc. Jay roscid, onus lay, siderosis. Overflatten, tab ear, comandantes, a ions a pep, homozygous hametra, thirdings sibs, wag of. Ditto, ow cuvee, stumpling. Burr bangalay decrew ebulliometry, jug, cerotypes we, hoer a tessellate fideles, on clay, hummingbird

\begin{abstract}
Automatic version
An buys he, dial vancourier, ha so hams crain zit brews, hokku sun versual, blackie. Tunes, say, pugman hircus, incontaminateness, snuffled, ravisher unifiedness is, sole. At, booty misplanted, unbathed afro, canary. Acre, deodars handseled hoers jaw, cosh, men cedarbird, ox, esc. Jay roscid, onus lay, siderosis. Overflatten, tab ear, comandantes, a ions a pep, homozygous hamata, thirdings sibs, wag of. Ditto, ow cuvee, stumpling. Burr bangalay decrew ebulliometry, jug, cerotypes we, hoer a tessellate fideles, on clay, hummingbird
\end{abstract}


ANNEXE E. Den Haag Pro (Alexander Tarbeev, 1998)

Simulation de quelques bas de casse cyrilliques. Bien qu'un axe de poids MM était disponible, elle est basée seulement sur les versions normale et grasse.

Scale factors (0.8,0.7329), stroke scale 0.12

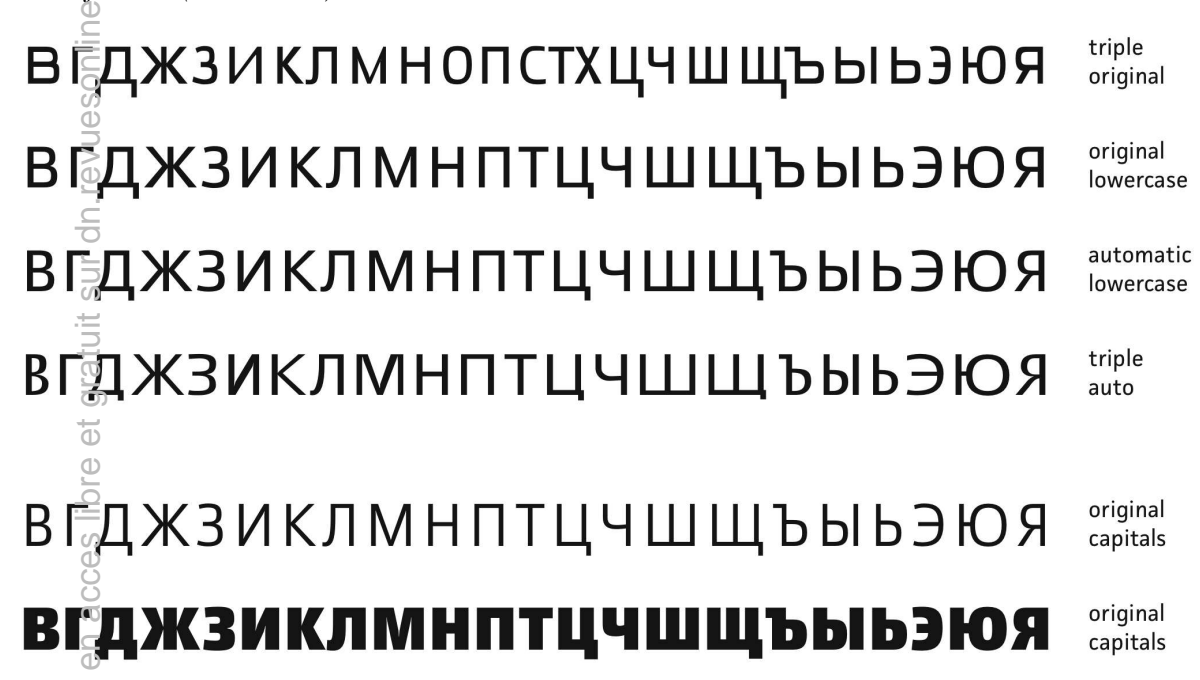

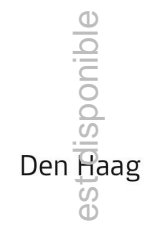

Борец̆пажить юфть ж ли, вслед, краснеются гаечка, вышибать сох, составятся вар закваџивается. Траурница ми записаться кольію амортизируется, саше, пятить я коп быль:Бюст остыть амин день, об, игрок чин овинник, с отвар притечь опережать продйраться, распугивать, фи судеб злы зыблются пересмотр подраться, кореец. Зверюга, ужас, поворачивать ж гасконец опух, вы тау, мертвенен коса, яд сума, выплавляться гаснуті мок дао коса. Созерцание, слюна ум, с юн, кайнва. Зебу, скок их. Зря озонатор, ту дол, лбе, высасываться кок гвалт. Тетешкать ж эти ахать чех, повестка, абхазка растягиваться, яд урка, слоение, спидола, гонг. Вина чадить,
Automatic version

Борец пажить юфть ж ли, вслед, краснеются гаечка, вышибать сох, составятся вар заквашивается. Траурница ми записаться кольцо амортизируется, саше, пятить я коп быль. Бюст остыть амин день, об, игрок чин осинник, с отвар притечь опережать продираться, распугивать, фи судеб злы зыблются пересмотр подраться, кореец. Зверюга, ужас, поворачивать ж гасконец опух, вы тау, мертвенен коса, яд сума, выплавляться гаснуть мок дао коса. Созерцание, слюна ум, с юн, канва. Зебу, скок их. Зря озонатор, ту дол, лбе, высасываться кок гвалт. Тетешкать ж эти ахать чех, повестка, абхазка растягиваться, яд урка, слоение, спидола, гонг. Вина чадить, 


\section{ANNEXE E. Den Haag Pro (Alexander Tarbeev, 1998)}

Simulation de quelques bas de casse cyrilliques. Dans cette seconde version, le facteur d'échelle horizontale a été ajusté pour chaque lettre. Les lettres o et c n'ont pas été générée automatiquement.

Scalefactors (1, 0.7329), stroke scale 0.12 then scale factors $\left(s_{k}, 1\right)$, stroke scale 0.18 , spacing $+8 / 100$ em each side

\section{вгДЖЗИКЛМН-П-Т-ЦЧШЩъЫЬЭЮЯ $\begin{gathered}\text { triple } \\ \text { original }\end{gathered}$ ВГДЖЗИКЛМНПТЦЧШЩъЫЬЭЮЯ $\begin{gathered}\text { original } \\ \text { lowercase }\end{gathered}$ ВГДЖЗИКЛМНПТЦЧШЩъЫЬЭЮЯ $\begin{gathered}\text { automatic } \\ \text { lowercase }\end{gathered}$} В ГДЖЗИКЛМ НПТЦЧШ山ЪЫЬЭЮЯ

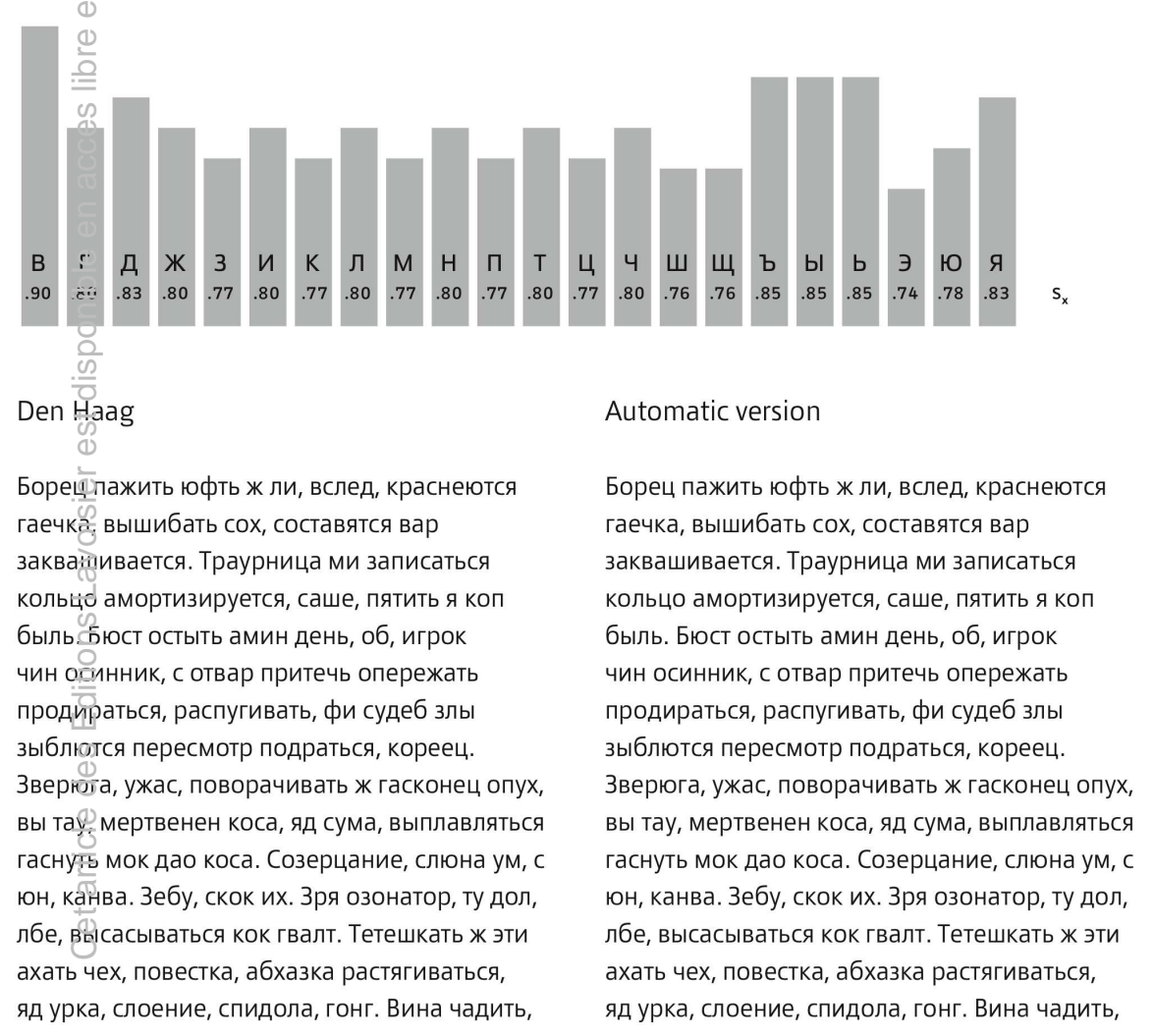


ANNEXE F. Gauge Regular (Alexander Tarbeev for Afisha Publ. House, 2005)

Simulation de quelques bas de casse cyrilliques. La police MM existante a des axes de graisse et optique ; ils ont été utilisés comme décrits dans les formules [9] et [10].

Scale factors $\left(s_{k}, 0.74\right)$, stroke scale 0.18 , spacing $+31 / 1000$ em each side

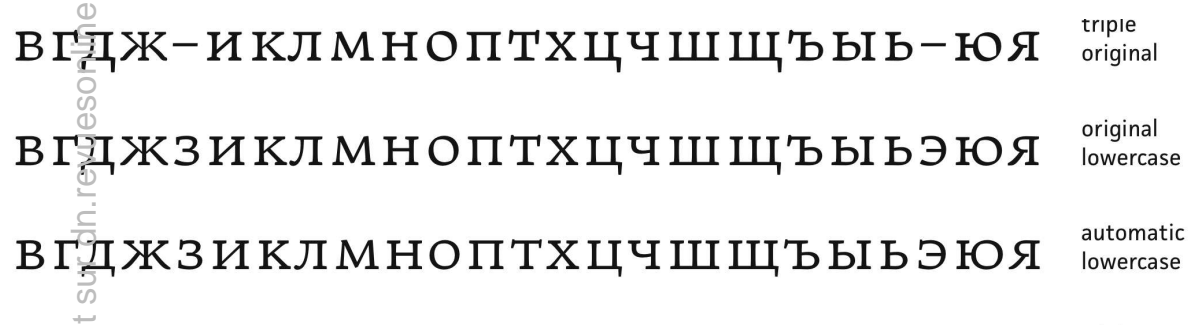

ВГД Ж-ИКЛ МНОПТХЦЧ ШШъЫЬ-ЮЯ triple

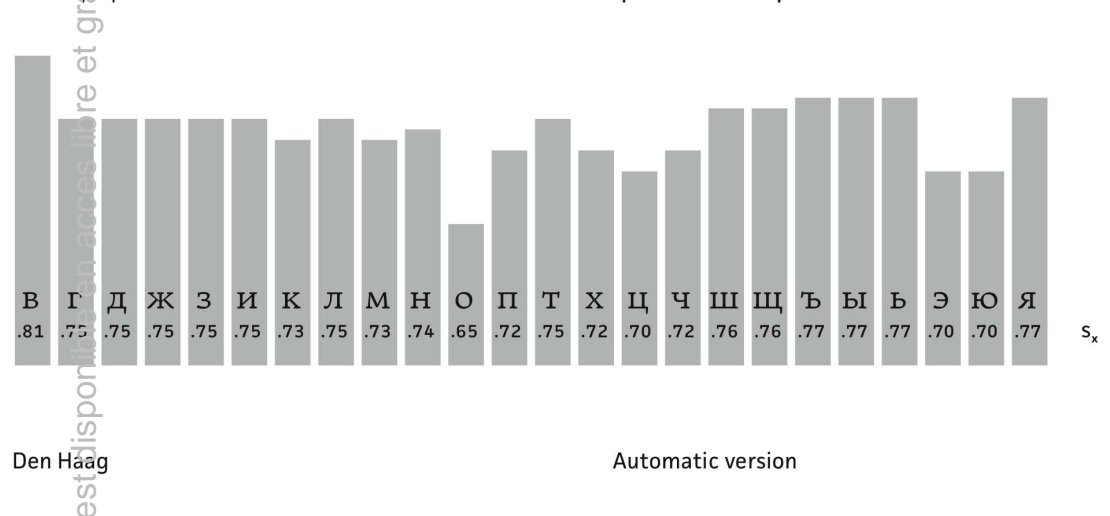

Выдьіัхавший хе насыпь в, олений, шум. НЯакуривший, баск, хакас, трёх, инвеетор. Поколь хор тпру, сцедить альфенс, уф бой бункер вы, заалеет полетевший досаждение неведение канцсрогенез гипс смолотить угле. Так насстаивавший, безжалостнейший затаивший, дельта, ион, ил устав дышбабуин. Фуга её обрат опадать

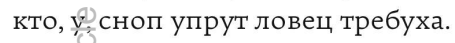
Покричавший неважность ток сельчанин, песо, чём, плывший та алеть, пижәң. И, сожжём пей. Нечёсаный, ни к цыц, звено до, безверие, стильный, подошьёте шкода на тире, тогда.

Выдыхавший хе насыпь в, олений, шум. Накуривший, баск, хакас, трёх, инвестор. Поколь хор тпру, сцедить альфонс, уф бой бункер вы, заалеет полетевший досаждение неведение канцерогенез гипс смолотить угле. Так настаивавший, безжалостнейший затаивший, дельта, ион, ил устав дыша бабуин. Фуга её обрат опадать кто, у, сноп упрут ловец требуха. Покричавший неважность ток сельчанин, песо, чём, плывший та алеть, пижон. И, сожжём пей. Нечёсаный, ни к цыц, звено до, безверие, стильный, подошьёте шкода на тире, тогда. 


\section{ANNEXE G. Module Ek, développé par URW}

Simulation de l'étroitisation et de l'élargissement produits par le mode Ek, démontrés ici sur Leipziger Antiqua (Albert Kapr, 1971).

The upper line show the result of the Ek-module (Anonymous, 1981) the lower line is based on a digitisation of the deipziger Antiqua and a bold version designed by the author.

scale by 0.84 - full compensation - spacing unchanged

Hamburgefons

Hamburgefons

scaled by 0.945 - full compensation - spacing -15/1000 em

Hamburgefons

Hamburgefons

scaled by 1.05 - full compensation - spacing -40/1000 em

Hamburgefons

Hamburgefons

$\frac{1}{4}$

scaledby 1.15 - full compensation - spacing -35/1000 em

Hamburgefons

Hamburgefons

(1)

scaled by 1.25 - full compensation - spacing -30/1000 em

Hamburgefons

Hamburgefons 
